\title{
Application of Urinary Polyphenol Biomarkers Measured by Liquid Chromatography Tandem Mass Spectrometry to Assess Polyphenol Intake and Their Association with Overweight and Obesity in Free-Living Healthy Subjects
}

\author{
Ruiyue Yang $\mathbb{D}^{D}$, Helu Xiu, Qi Zhou, Liang Sun ${ }^{D}$, Hongna Mu, Hongxia Li, Siming Wang, \\ Xianghui Li, Wenxiang Chen, and Jun Dong
}

The MOH Key Laboratory of Geriatrics, Beijing Hospital, National Center of Gerontology, Beijing 100730, China

Correspondence should be addressed to Ruiyue Yang; ruiyue_yang@163.com and Jun Dong; jun_dong@263.net

Received 10 March 2019; Accepted 12 May 2019; Published 16 June 2019

Guest Editor: Fangyi Xu

Copyright (C) 2019 Ruiyue Yang et al. This is an open access article distributed under the Creative Commons Attribution License, which permits unrestricted use, distribution, and reproduction in any medium, provided the original work is properly cited.

\begin{abstract}
Although some polyphenol biomarkers in serum or urine have been identified by untargeted metabolomics and proved to reflect dietary polyphenol intake, only a few of them have been validated in different studies and populations with simple and reliable targeted methods. In the present study, a targeted metabolomics method by LC/MS/MS for the measurement of twenty-two polyphenol biomarkers in urine samples was established and validated to effectively assess the habitual polyphenol intake in free-living healthy Chinese subjects. Multivariate logistic regression models were used to assess relationships of biomarkers with overweight and obesity after adjusting for potential confounders. The levels of urinary polyphenol biomarkers, especially gut microbial metabolites of polyphenols, were inversely associated with overweight and obesity, and this association was more pronounced in the inflammatory groups, suggesting that it is of great importance to maintain polyphenol biomarkers at high levels or intake-sufficient polyphenols in obesity with chronic inflammation than others. The measurement of these biomarkers may offer a valid alternative or complementary addition to self-reported survey for the evaluation of polyphenol intake and investigation into their relationships with chronic disease-related endpoints in large-scale clinical and epidemiologic studies.
\end{abstract}

\section{Introduction}

Polyphenols composed of a wide variety of phytochemicals abundant in plants, ranging from simple phenolic acids to flavonoid polymers, are commonly consumed through a diet rich in fruit, vegetables, tea, wine, and soy-based foods [1]. Habitual consumption of dietary polyphenols has been consistently linked with a wide spectrum of potential health benefits in humans, including anti-inflammatory, antiobesity, gastroprotective, and anticarcinogenic effects [2-4].

Despite advances in nutritional epidemiological study design and analytical strategies, precise estimation of polyphenol intake in free-living populations remains a major challenge [5], because polyphenols are abundant, wide-spanning, and diverse in the human diet, and their quantity in foods is heavily influenced by a food's growth and processing conditions [6]. Most epidemiologic studies rely on selfreported dietary assessment methods that might suffer from systematic and random errors related to the variability in the polyphenol content of foods, bias in dietary misreporting (especially underreporting of bias towards unhealthy foods and overreporting of bias towards fruits and vegetables), and differences in absorption and metabolism between individuals [7-9]. Moreover, underreporting of intake of unhealthy foods is particularly common in an obesity population [10], which is a major concern considering the increasing prevalence of obesity worldwide.

Therefore, there is a pressing need for dietary polyphenol biomarkers to precisely evaluate polyphenol intake. Dietary biomarkers have the potential to improve the measurement of dietary exposures by validating diet questionnaires [11] or by replacing inadequate dietary data [12]. Metabolomics, 
the measurement of small molecules, including metabolic parent compounds, substrates, and products, in biospecimens, may more precisely quantify dietary exposures and thus provide better estimates of disease risk in epidemiologic studies [13]. Metabolites may better reflect "true exposure" and also capture exposure to nonnutritive substances, such as compounds generated by cooking or gut microbiota, which may play important roles in disease etiology $[14,15]$.

Recent studies have shown that metabolomics can be successfully applied to dietary research and can identify some novel potential dietary polyphenol biomarkers, but most studies were small dietary interventions [16-19]. Although most of these studies calculated the half-life using single doses of polyphenols or polyphenol-rich foods, in fact, the intake of polyphenol is usually chronic; thus, biomarkers identified may not perform well as proxies for usual food intake $[7,14]$. A case in point is that a recent citrus feeding study identified more than 600 metabolites associated with acute citrus consumption; however, only 12 metabolites were related to regular dietary citrus consumption in a free-living population [19].

Considering that polyphenols or polyphenol-rich foods are generally consumed almost daily, it would be advantageous to identify biomarkers of chronic intakes and apply these biomarkers in epidemiological studies, with chronic nutritional doses, using the usual biofluids collected in epidemiological studies, such as plasma or morning urine. A recent untargeted metabolomics study proved that metabolic profiles of urine samples developed in a highly controlled environment reflect dietary intake and can be used to model and classify dietary patterns of free-living populations [20].

The untargeted metabolomics could discover new biomarkers and often offer qualitative concentrations (i.e., relative concentration), while the targeted metabolomics allows us to obtain a "real" quantification (i.e., absolute concentration) of these biomarkers. Accordingly, these biomarkers can be easily applied in epidemiological studies. Despite the relatively high number of polyphenol biomarkers, only a few of them have been validated in different studies and populations with simple and reliable targeted methods. Most of the related studies mainly involved North American and European people. Thus, the relationships need to be further validated in external independent populations, especially in Asian people of whose category and content of foods for dietary polyphenol intake and food cooking methods are very different from those in the West.

Therefore, we proposed to use a cross-sectional study of free-living Chinese subjects to investigate and validate the effect of urinary polyphenol biomarkers measured by liquid chromatography tandem mass spectrometry (LC/MS/MS) on the assessment of polyphenol intake and their association with overweight and obesity.

\section{Materials and Methods}

2.1. Study Population. This is a cross-sectional study conducted on Chinese subjects. A total of 123 apparently healthy participants (52 males and 71 females, $42.3 \pm 15.2$ years old) were recruited at random from a group of Beijing residents attending an annual physical examination at Beijing Hospital in December 2013. Participants were excluded if they had malignant tumors, blood system diseases, chronic obstructive pulmonary disease, autoimmune diseases, infectious diseases, or other clinically significant illnesses, if they were taking prescription medication or nutritional supplements, or if they presented any abnormalities on physical examination, electrocardiography, or screening blood tests. Women were ineligible if they were pregnant or breastfeeding. The participants were required to complete a questionnaire with the help of an investigator. The sera from fasting blood samples obtained from subjects were isolated and stored at $-80^{\circ} \mathrm{C}$ until being analyzed. The first-void urine samples were collected in $0.5 \% \mathrm{HCl}$, and after centrifugation, ten $600 \mu \mathrm{L}$ aliquots were stored at $-80^{\circ} \mathrm{C}$ before analysis. This study was approved by the Ethics Committee of the Beijing Hospital of the Ministry of Health, and written informed consents were obtained from all participants.

2.2. Measurement of Urinary Total Polyphenol and 22 Polyphenol Biomarkers. Total polyphenols in urine (uTP) were measured by the Folin-Ciocalteu method modified by Medina-Remon et al. [21]. Briefly, urine samples were applied to the Plexa PAX solid phase extraction cartridge; after washing, the polyphenols were eluted, evaporated, and reconstituted. Total polyphenols were measured by FolinCiocalteu colorimetric assay, calculated by gallic acid standard curve, and corrected by urine creatinine levels. uTP was expressed as mg gallic acid equivalent (GAE)/g of Crea.

A targeted metabolomics method by LC/MS/MS was developed and applied to the measurement of 22 polyphenol biomarkers in urine samples. Standards of 22 polyphenol biomarkers, HPLC grade acetonitrile, and ammonium acetate were obtained from Sigma-Aldrich (St. Louis, MO, USA). Generally, aliquots of $500 \mu \mathrm{L}$ calibrators and urine samples were respectively applied to the Plexa PCX solid phase extraction cartridge. After extraction and purification, the reconstituted solutions were injected into an LC/MS/MS system. LC-MS/MS analysis was performed using an Applied Biosystems Sciex API 5500 QTRAP tandem mass spectrometer (Framingham, MA, USA) equipped with an Agilent 1260 Series HPLC system (Santa Clara, CA, USA). The LC separation was performed by an XTerra MS C18 $(2.1 \mathrm{~mm} \times 100 \mathrm{~mm}, 3.5 \mu \mathrm{m})$ column. The injection volume was $10 \mu \mathrm{L}$, and the flow rate was $300 \mu \mathrm{L} / \mathrm{min}$. Gradient elution was carried out with $5 \mathrm{mmol} / \mathrm{L}$ ammonium acetate as mobile phase $\mathrm{A}$ and acetonitrile as mobile phase $\mathrm{B}$. We applied a gradient profile with the following proportions $(v / v)$ of phase B $[t(\mathrm{~min}), \% \mathrm{~B}]:(0,0),(5,15),(8,50),(9$, $100),(14,100)$, and $(15,0)$. The column was reequilibrated for $20 \mathrm{~min}$. MS detection was performed with negative electronic spray ionization (ESI) in a multiple reaction monitoring (MRM) mode at a source temperature of $600^{\circ} \mathrm{C}$ and a voltage of $-4000 \mathrm{~V}$. The dwell times were $0.08 \mathrm{~s}$ for MRM. Nitrogen was used as the curtain, nebulizer, and collision gas at pressures of 30,80 , and 80 psi, respectively. Ion transition of each polyphenol biomarker was presented in Supplementary Table 3). The calibration curve was established using a regression of peak areas $(y)$ of each 
polyphenol to the corresponding concentrations $(x)$ of the calibrators. The calculated concentrations of the polyphenols were corrected by urine Crea levels. And by using the PCX extraction column, most of the 22 polyphenols had recoveries of $>80 \%$. All polyphenols were separated and analyzed within $14 \mathrm{~min}$. Imprecision analysis showed that the established LC/MS/MS method had intra-assay and total imprecisions of $1.00 \% \sim 10.62 \%$ and $3.70 \% \sim 17.09 \%$, respectively.

2.3. Dietary and Covariate Assessment. Covariate data of demographic and lifestyle variables were ascertained by a questionnaire. Usual dietary intake over the 6 months before the survey was measured by a short self-administered food frequency questionnaire (FFQ). The survey provided information on frequency of consumption and category of polyphenol-rich foods in Chinese dietary habits. The levels of consumption of fruits, vegetables, coffee, and tea were categorized as binary (low or high). The levels of polyphenol content in various consumption fruits were estimated by the Phenol-Explorer 3.6 database [22] and categorized as binary [low (bottom and middle tertiles) or high (top tertile)]. Subjects with four or more of the five dietary conditions, including consumption of high levels of fruits, vegetables, coffee, or tea and high level of polyphenol content of consumption fruits, were assigned to the polyphenol-rich diet group.

Height, body weight, and sitting blood pressure (BP) were measured. The serum samples were tested for fasting blood glucose (FBG), total cholesterol (TC), triglyceride (TG), high-density lipoprotein cholesterol (HDL-C), lowdensity lipoprotein cholesterol (LDL-C), and C-reactive protein (CRP), using assay kits from Sekisui Medical Technologies (Osaka, Japan) on a Hitachi 7180 chemistry analyzer. Serum concentrations of 8-isoprostane (IsoP) were determined by the commercial EIA kit (Cayman Chemical Company, USA). The diagnoses of overweight/obesity and other components of MetS were made according to the criteria provided by the Chinese Diabetes Society (2004) [23].

2.4. Statistical Analysis. The main continuous variables were all tested for normality. Normally distributed data were expressed as means $\pm S D$, whereas variables obeying a skewed distribution were shown as the median (interquartile range). Count data were reported as frequencies and percentages. We constructed the mean SD score ( $z$ score) [24] of 22 urinary polyphenol biomarkers for subjects with complete data to reduce the large number of correlated variables into uncorrelated factors [25]. The $z$ score of 22 urinary polyphenol biomarkers was computed by the summary of $z$ score of each biomarker/22. For quantitative data, the variance analysis was conducted to compute the difference across the tertile of the $z$ score of urinary polyphenol biomarkers, whereas for count data, the chi-square test was used. Correlation coefficients between levels of $z$ scores of each urinary polyphenol biomarker and metabolic traits were calculated by Spearman's correlation. The nonparametric Mann-Whitney test was used to evaluate the differences of $z$ scores of urinary polyphenol biomarkers between two diet groups or the nor- mal and overweight/obesity groups. Principal component analysis (PCA) was also conducted for 22 urinary polyphenol biomarkers to determine the variation in attributes between polyphenol-rich and normal diet groups. Multivariate logistic regression models were used to estimate the odds ratios (ORs) and 95\% confidence intervals (CIs), which adopted the entry method and the maximum likelihood ratio test for overweight/obesity. Potential confounding variables, including age, gender, FBG, UA, CREA, CRP, IsoP, hypertension, dyslipidemia, smoking and alcohol drinking status, high levels of physical activity, and polyphenol-rich diet were controlled in the regression models. The performance of the $z$ score of urinary polyphenol biomarkers for classifying overweight/obesity was assessed by logistic regression and receiver operating characteristic analysis. Stratified and interaction analyses were performed according to serum CRP levels $[<0.7$ (median) and $\geq 0.7 \mathrm{mg} / \mathrm{L}]$. To further explore the nonlinear interaction of polyphenol biomarkers and serum CRP concentration for overweight/obesity, we treated the serum CRP by restricted cubic splines (RCS) with three freedom degrees in the age- and genderadjusted logistic models. Two-sided $p$ values $<0.05$ were considered statistically significant. SPSS 21.0 software (SPSS Inc.) was used for the statistical analysis of the data, and $\mathrm{R}$ packages of "vegan 2.5-4" and "splines 0.2.8" were used for PCA and RCS analysis.

\section{Results}

3.1. Characteristics of Population according to the Levels of Urinary Polyphenol Biomarkers. The concentration of 22 urinary polyphenol biomarkers of 123 subjects had skewed distribution, and they could not be normally distributed after natural logarithm, logarithmic transformation of 10 , and square root (SQRT) transformation. The prevalence of overweight and obesity in our study was $28.5 \%$. As shown in Table 1, according to the tertile of the $z$ score of 22 urinary polyphenol biomarkers, the subjects with higher levels of urinary polyphenol biomarkers were more likely to be older and female ( $p$ trend $=0.014$ and 0.008 , respectively). With respect to metabolic traits, HDL-C significantly increased with increasing urinary polyphenol biomarker levels, which had a dose-response relationship $(p=0.017$ for trend); in contrast, UA and CREA levels declined notably ( $p$ trend $=0.010$ and 0.030 , respectively). Additionally, with increasing urinary polyphenol biomarker levels, subjects tended to have habits of high consumption of fruits, tea, and polyphenol-rich diet (all $p$ trend $<0.05$ ) and have fewer current drinkers ( $p$ trend $=0.031$ ). Expectably, the concentration of urinary total polyphenol by FolinCiocalteu colorimetric assay increased gradually with increment levels of the $z$ score of 22 urinary polyphenol biomarkers ( $p$ for trend $<0.001$ ).

3.2. Correlations between Levels of Urinary Polyphenol Biomarkers and Cardiometabolic Risk Factors. As shown in Figure 1, Spearman's correlation analysis demonstrated the correlation between metabolic characteristics and levels of urinary polyphenol biomarkers. Most of the urinary 
TABLE 1: Characteristics of the study participants by the $z$ score of 22 urinary polyphenol biomarkers.

\begin{tabular}{|c|c|c|c|c|}
\hline \multirow{2}{*}{ Characteristic $^{\mathrm{a}}$} & \multicolumn{3}{|c|}{ Tertile of $z$ score of 22 urinary polyphenol biomarkers ${ }^{\mathrm{b}}$} & \multirow{2}{*}{$p$ trend $^{c}$} \\
\hline & $\mathrm{T} 1$ & $\mathrm{~T} 2$ & T3 & \\
\hline Number & 41 & 41 & 41 & \\
\hline Age (y) & $36.3 \pm 14.3$ & $46.4 \pm 14.2$ & $44.3 \pm 15.5$ & 0.014 \\
\hline Male, $n(\%)$ & $24(58.5)$ & $16(39.0)$ & $12(29.3)$ & 0.008 \\
\hline BMI $\left(\mathrm{kg} / \mathrm{m}^{2}\right)$ & $23.0 \pm 3.6$ & $23.7 \pm 3.4$ & $22.4 \pm 2.5$ & 0.425 \\
\hline SBP (mmHg) & $112.2 \pm 16.4$ & $119.0 \pm 15.6$ & $114.6 \pm 13.3$ & 0.482 \\
\hline DBP (mmHg) & $73.4 \pm 10.3$ & $76.6 \pm 7.5$ & $74.1 \pm 6.9$ & 0.711 \\
\hline FBG $(\mathrm{mmol} / \mathrm{L})$ & $5.0 \pm 0.5$ & $5.1 \pm 0.6$ & $4.9 \pm 0.5$ & 0.618 \\
\hline $\mathrm{TG}(\mathrm{mmol} / \mathrm{L})$ & $1.0(0.8 \sim 1.5)$ & $1.0(0.6 \sim 1.5)$ & $0.9(0.6 \sim 1.3)$ & 0.104 \\
\hline $\mathrm{TC}(\mathrm{mmol} / \mathrm{L})$ & $4.5 \pm 0.9$ & $4.9 \pm 1.1$ & $4.6 \pm 0.8$ & 0.646 \\
\hline HDL-C (mmol/L) & $1.3(1.1 \sim 1.5)$ & $1.4(1.3 \sim 1.7)$ & $1.5(1.2 \sim 1.8)$ & 0.017 \\
\hline LDL-C (mmol/L) & $2.6(2.3 \sim 3.4)$ & $3.1(2.4 \sim 3.9)$ & $2.7(2.3 \sim 3.4)$ & 0.911 \\
\hline $\mathrm{UA}(\mu \mathrm{mol} / \mathrm{L})$ & $309.4 \pm 76.8$ & $281.5 \pm 62.8$ & $268.5 \pm 72.0$ & 0.010 \\
\hline CREA $(\mu \mathrm{mol} / \mathrm{L})$ & $69.1 \pm 13.6$ & $63.8 \pm 11.9$ & $62.4 \pm 16.0$ & 0.030 \\
\hline Overweight/obesity ${ }^{\mathrm{d}}, n(\%)$ & $13(31.7)$ & $16(39.0)$ & $6(14.6)$ & 0.088 \\
\hline Hypertension $^{\mathrm{d}}, n(\%)$ & $6(14.6)$ & $4(9.8)$ & $3(7.3)$ & $0.373^{\mathrm{e}}$ \\
\hline Dyslipidemia $^{\mathrm{d}}, n(\%)$ & $8(19.5)$ & $7(17.1)$ & $8(19.5)$ & 1.000 \\
\hline Impaired fasting glucose ${ }^{\mathrm{d}}, n(\%)$ & $1(2.4)$ & $3(7.3)$ & $2(4.9)$ & $0.802^{\mathrm{e}}$ \\
\hline $\mathrm{MetS}^{\mathrm{d}}, n(\%)$ & $3(7.3)$ & $0(0)$ & $0(0)$ & - \\
\hline Current smoker, $n(\%)$ & $8(19.5)$ & $4(9.8)$ & $9(22.0)$ & $0.884^{\mathrm{e}}$ \\
\hline Current drinker, $n(\%)$ & $14(34.1)$ & $6(14.6)$ & $6(14.6)$ & 0.031 \\
\hline High levels of physical activity, $n(\%)$ & $15(37.5)$ & $17(41.5)$ & $17(41.5)$ & 0.719 \\
\hline High consumption of vegetables, $n(\%)$ & $20(48.8)$ & $23(56.1)$ & $26(63.4)$ & 0.184 \\
\hline High consumption of fruits, $n(\%)$ & $11(26.8)$ & $18(43.9)$ & $29(70.7)$ & $<0.001$ \\
\hline $\begin{array}{l}\text { High levels of polyphenol content of } \\
\text { consumption fruits, } n(\%)\end{array}$ & $15(36.6)$ & $14(34.1)$ & $16(39.0)$ & 0.819 \\
\hline High consumption of coffee, $n(\%)$ & $3(7.3)$ & $2(4.9)$ & $2(4.9)$ & $0.815^{\mathrm{e}}$ \\
\hline High consumption of tea, $n(\%)$ & $6(14.6)$ & $11(26.8)$ & $14(34.1)$ & 0.043 \\
\hline Polyphenol-rich diet, $n(\%)$ & $7(17.1)$ & $10(24.4)$ & $19(46.3)$ & 0.004 \\
\hline CRP (mg/L) & $0.6(0.3 \sim 1.1)$ & $0.8(0.5 \sim 1.6)$ & $0.5(0.2 \sim 1.2)$ & 0.609 \\
\hline IsoP (ng/L) & $92.4(57.8 \sim 138.1)$ & $117.0(50.0 \sim 164.1)$ & $83.9(45.3 \sim 123.1)$ & 0.459 \\
\hline uTP (mg GAE/g Crea) & $77.4(63.7 \sim 98.4)$ & $117.8(95.4 \sim 136.7)$ & $145.8(120.0 \sim 211.1)$ & $<0.001$ \\
\hline
\end{tabular}

BMI: body mass index; SBP: systolic blood pressure; DBP: diastolic blood pressure; FBG: fasting blood glucose; TG: triglyceride; TC: total cholesterol; HDL-C: high-density lipoprotein cholesterol; LDL-C: low-density lipoprotein cholesterol; UA: uric acid; CREA: creatinine; MetS: metabolic syndrome; CRP: C-reactive protein; IsoP: 8-isoprostane; uTP: urinary total polyphenol. ${ }^{a}$ Data are the mean $\pm \mathrm{SD}$, median (interquartile range) for continuous variables, or cases (percentage) for categorical variables. ${ }^{\mathrm{b}}$ The $z$ score of 22 urinary polyphenol biomarkers was computed by the summary of the $z$ score of each biomarker/22. ${ }^{c} p$ values for trend. ${ }^{\mathrm{d} D e f i n e d ~ a c c o r d i n g ~ t o ~ t h e ~ c r i t e r i a ~ o f ~ m e t a b o l i c ~ s y n d r o m e ~ f r o m ~ C h i n e s e ~ D i a b e t e s ~ S o c i e t y ~(C D S) ~(2004) ~ f o r ~ C h i n e s e . ~}$ ${ }^{\mathrm{e}} p$ values for the Fisher exact test.

polyphenol biomarkers showed remarkable positive correlation with urinary total polyphenol and age. Significantly negative correlations were found respectively between most of urinary polyphenol biomarkers, especially gut microbial metabolites, and cardiovascular risk factors, such as TC, TG, LDL-C, FBG, UA, CRP, and IsoP $(p<0.05)$, while positive correlation existed between these biomarkers and HDL-C, respectively.

3.3. Comparison of Levels of Urinary Polyphenol Biomarkers according to the Presence of Polyphenol-Rich Diet. As shown in Figure 2(a), subjects with polyphenol-rich dietary habit have higher levels of most of urinary polyphenol biomarkers compared with the normal diet group. Ferulic acid $(p=0.048)$, coumaric acid $(p=0.048)$, syringic acid $(p=0.008)$, kaempferol $(p=0.029), \quad(3,4)$-dihydroxyphenylacetic acid $(p=0.006)$, sinapic acid $(p=0.004)$, hippuric acid $(p=0.044)$, and $z$ score of the total 22 urinary polyphenols $(p=0.004)$ levels in the polyphenol-rich diet group were significantly increased in comparison with the other groups.

Principal component analysis and biplot (Figure 3) showed that 22 urinary polyphenol biomarkers can differentiate effectively the polyphenol-rich diet from the other one. Six of urinary polyphenol biomarkers, including 


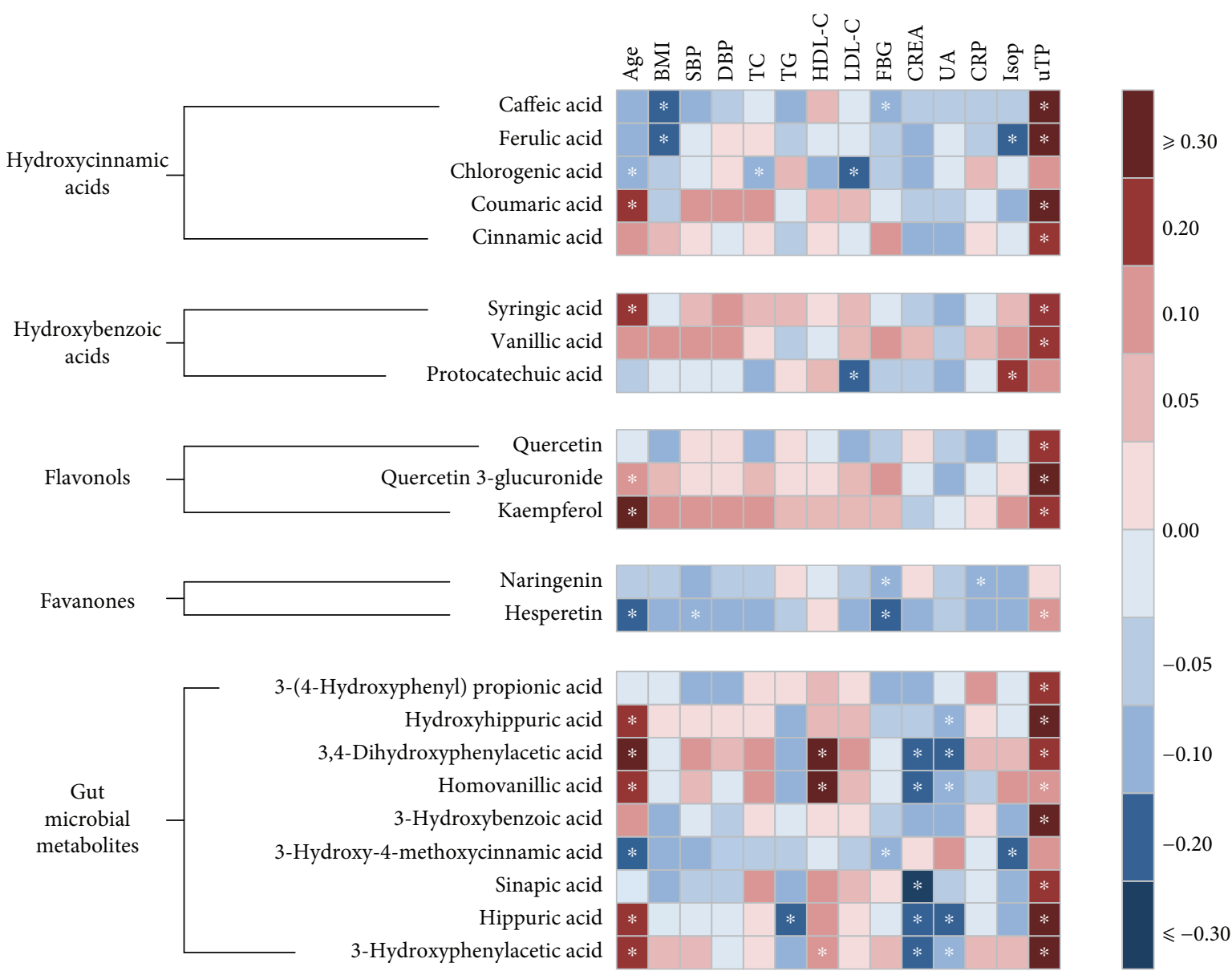

FIGURE 1: Correlation heatmap illustrating the relationship between urinary polyphenol biomarkers and cardiometabolic quantitative phenotypes in the study population. Spearman's correlation coefficients are presented in a blue-white-red color scheme. Dark red indicates a more positive correlation, and dark blue indicates a more negative correlation; white indicates no correlation. ${ }^{*} p<0.05$. BMI: body mass index; SBP: systolic blood pressure; DBP: diastolic blood pressure; FBG: fasting blood glucose; TG: triglyceride; TC: total cholesterol; HDL-C: high-density lipoprotein cholesterol; LDL-C: low-density lipoprotein cholesterol; UA: uric acid; CREA: creatinine; CRP: C-reactive protein; IsoP: 8-isoprostane; uTP: urinary total polyphenol.

ferulic acid, hydroxyhippuric acid, sinapic acid, hippuric acid, 3-hydroxyphenylacetic acid, and 3-(4-hydroxyphenyl) propionic acid, contributed the most to distinguishing the two diet groups.

3.4. Association of Levels of Urinary Polyphenol Biomarkers with Overweight/Obesity. According to the definition of MetS, the population was divided into the control and case groups, respectively, based on whether the population had MetS, overweight/obesity, hypertriglyceridemia, dyslipidemia, and impaired fasting glucose. Regarding the patterns of dichotomous cardiometabolic traits, as shown in Figure 2(b), overweight and obesity subjects have lower levels of most of urinary polyphenol biomarkers compared with the normal group. Caffeic acid $(p=0.002)$, ferulic acid ( $p=0.002)$, 3-hydroxybenzoic acid $(p=0.040)$, and $z$ score of the total 22 urinary polyphenol $(p=0.047)$ levels in the overweight and obesity groups were significantly decreased in comparison with the normal group. There are no statistical differences of $z$ score of urinary polyphenol biomarkers between different groups according to the presence of MetS and other components of MetS.

Furthermore, the associations between the levels of urinary polyphenol biomarkers and overweight/obesity were analyzed by multivariate logistic regression (Table 2). After adjusting for age, gender, FBG, UA, CREA, CRP, IsoP, hypertension, dyslipidemia, smoking and alcohol drinking status, high levels of physical activity, and polyphenol-rich diet, the ORs for overweight/obesity were all meaningful no matter whether $z$ score levels of urinary polyphenol biomarkers were regarded as continuous variables or categorical variables (all $p<0.05$ ). Each 1 score increase of $z$ score levels of urinary polyphenol biomarkers was associated with approximately a 28 -fold decreased risk of overweight/obesity [OR (95\% CI): 0.036 (0.004 0.358), $p=0.005]$. Besides, tertile analyses revealed linear decreases of overweight/obesity risk with an approximately 8 -fold decrease in the risk of overweight/obesity in the top vs. bottom tertile of $z$ score levels of urinary polyphenol biomarkers [OR (95\% CI): 0.133 (0.027 0.666), $p$ trend $=0.015]$. 


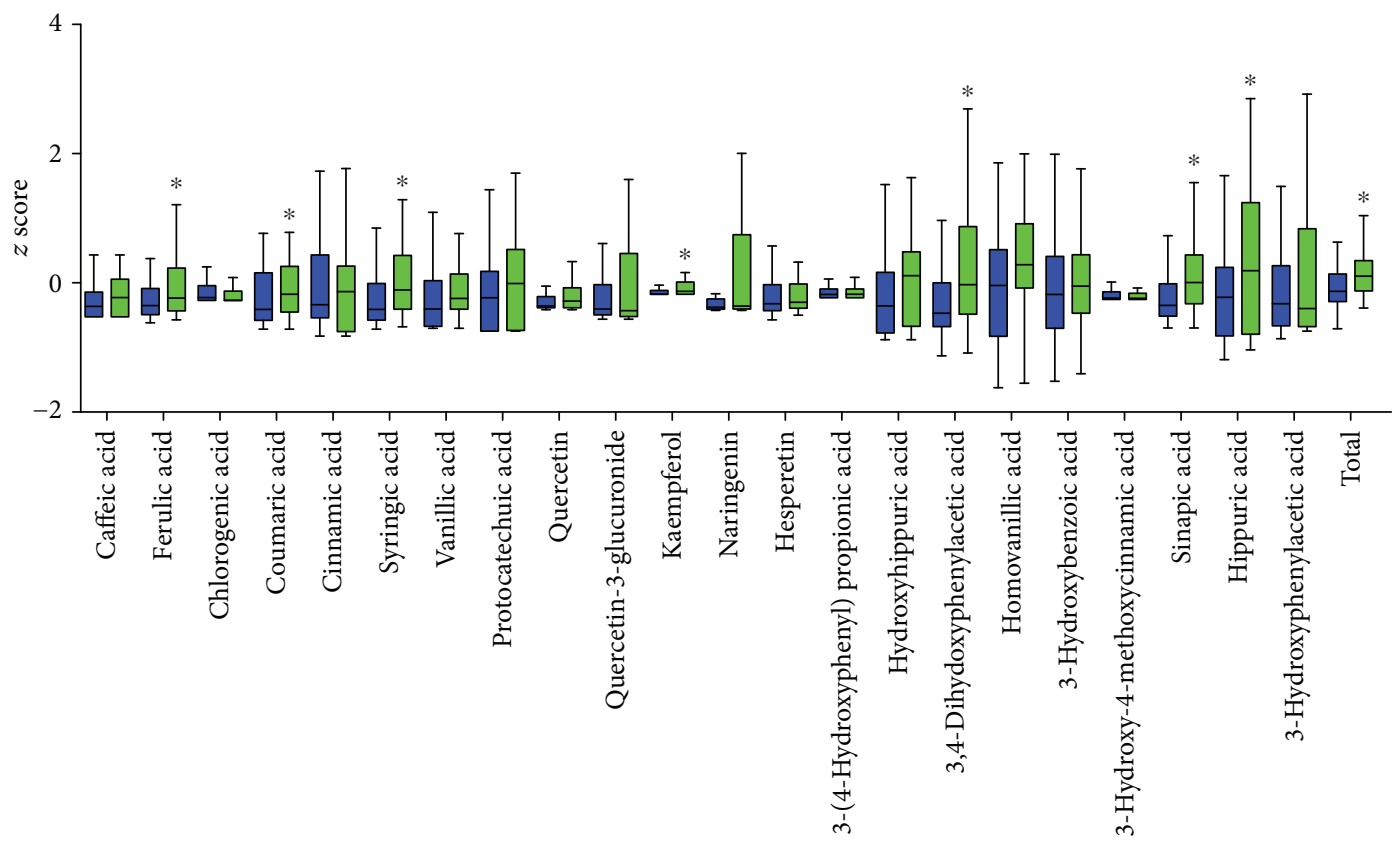

Normal diet

$\square$ Polyphenol-rich diet

(a)

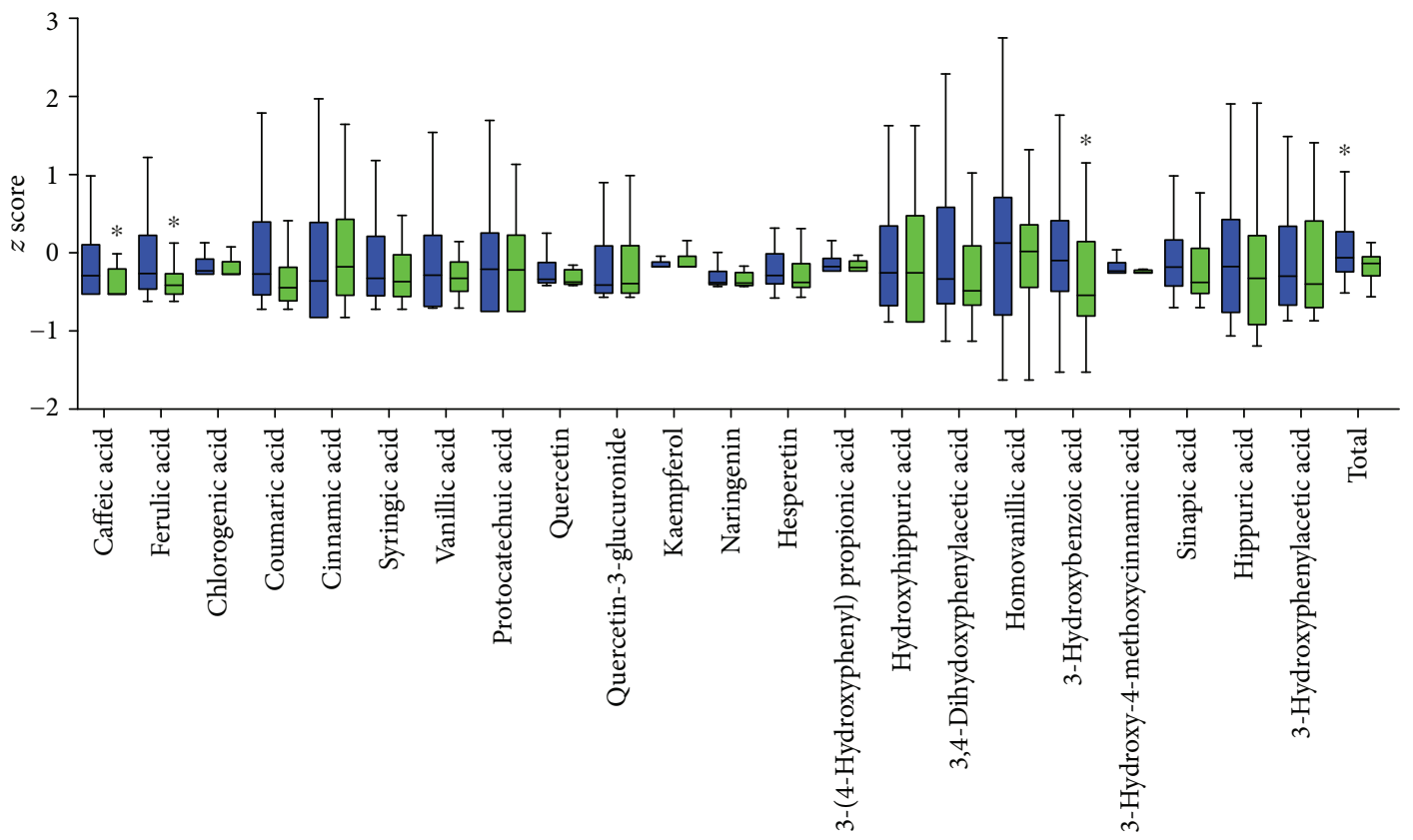

$\square$ Normal

$\square$ Overweight/obesity

(b)

FIGURE 2: Comparison of $z$ scores of concentration of urinary polyphenol biomarkers according to the presence of (a) polyphenol-rich diet or (b) overweight/obesity. ${ }^{*} p<0.05$. Data are depicted by box plots extending from the 25 th to the 75 th percentile and whiskers ranging from the lower limit to the upper limit. The horizontal line in the box plot represents the median. The $z$ score of total 22 polyphenol biomarkers was computed by the summary of the $z$ score of each polyphenol biomarker/22.

Besides, by means of making an analysis of the ORs of each type of polyphenol biomarkers for overweight/obesity, we observed that urinary levels of gut microbial metabolites of polyphenol had the most remarkable correlation with overweight/obesity [OR (95\% CI): 0.099 (0.019 0.006), p trend $=0.015]$ (Table 3). 


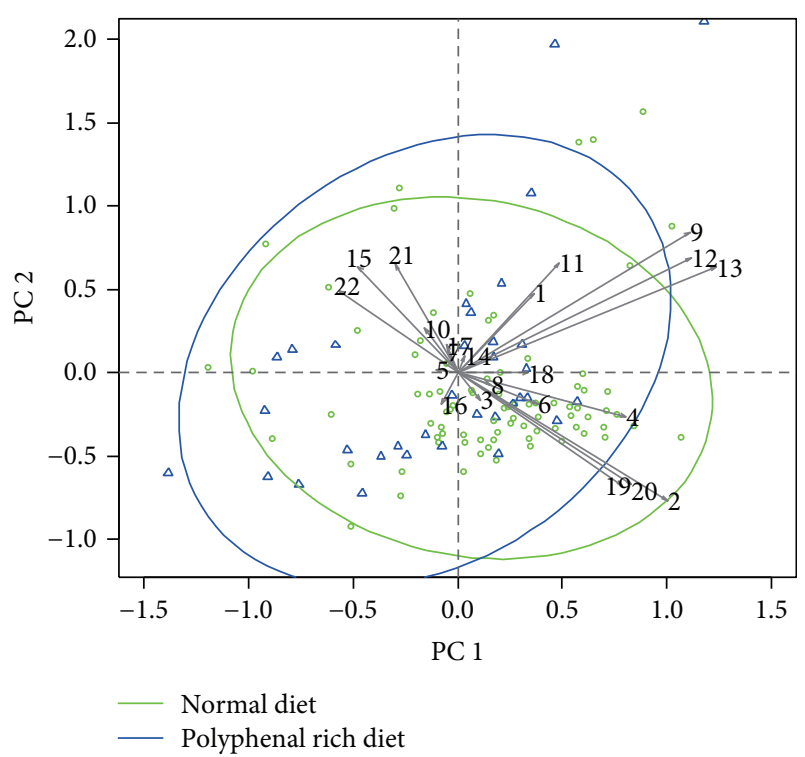

Figure 3: Principal component analysis plot for polyphenol-rich and normal diets with urinary polyphenol biomarkers. Arabic numerals of 1-22 represent caffeic acid, ferulic acid, chlorogenic acid, coumaric acid, cinnamic acid, syringic acid, vanillic acid, protocatechuic acid, quercetin, quercetin 3-glucuronide, kaempferol, naringenin, hesperetin, (3,4)-dihydroxyphenylacetic acid, hydroxyhippuric acid, 3-hydroxybenzoic acid, homovanillic acid, 3-hydroxy-4-methoxycinnamic acid, sinapic acid, hippuric acid, 3-hydroxyphenylacetic acid, and 3-(4-hydroxyphenyl) propionic acid, respectively. The ellipses represent the $80 \%$ confidence intervals.

\subsection{More Significant Association between Urinary Polyphenol} Biomarker Levels and Overweight/Obesity in the Inflammatory Groups. The stratified analysis of the associations of urinary polyphenol biomarker levels and overweight/obesity was conducted according to a median concentration $(0.7 \mathrm{mg} / \mathrm{L})$ cut-off point of CRP. The results showed that the OR (95\% CI) between urinary polyphenol biomarker levels and overweight/obesity was 0.000067 (0.00-0.017) in those participants with higher CRP levels, whereas in the lower CRP group, the OR (95\% CI) was $0.425(0.0069-29.547)$, in a multivariable logistic model as mentioned above. A significant interaction was observed between urinary polyphenol biomarker levels and CRP for overweight/obesity ( $p$ for interaction $=0.033$ ). Furthermore, we used restricted cubic splines to explore the nonlinear interaction of polyphenol biomarkers and serum CRP concentration for overweight/obesity. In Figure 4, with the increase of serum CRP concentration, the inverse relationship of polyphenol biomarkers with overweight/obesity gradually enhanced, until reaching a state of almost infinitely large.

3.6. Assessment of ROC Curves for Overweight/Obesity. We used ROC analysis to assess the performance of the classifier models for overweight/obesity classification. ROC curves and measures of the area under the curve (AUC) were presented for three multivariable models: (1) clinical model inclusive of traditional risk factors (age, gender, FBG, UA, CREA, CRP, IsoP, hypertension, dyslipidemia, smoking and alcohol drinking status, and physical activity); (2) model 1 plus polyphenol-rich diet; and (3) model 2 plus the $z$ score of 22 urinary polyphenol biomarkers. In Figure 5, the results showed greater discriminative capability for the clinical model containing urinary polyphenol biomarkers (AUC 0.884, 95\% CI: 0.813 0.956) compared with the model containing clinical variables only (AUC 0.846, 95\% CI: $0.775 \sim 0.917$ ). The model of clinical factors containing urinary polyphenol biomarkers yielded a sensitivity of $82.8 \%$ and a specificity of $88.2 \%$.

\section{Discussion}

In the present study, twenty-two polyphenol biomarkers were simultaneously quantified by LC/MS/MS analysis using a reverse-phase column and multiple reaction monitoring detection. These biomarkers, including hydroxycinnamic acids, hydroxybenzoic acids, flavonols, favanones, and gut microbial metabolites, were selected for their native forms abundant in Chinese dietary habits, for being representative of the main types of polyphenol metabolites reported to correlate with polyphenol intake by previous studies, and for being commercially available, on the basis of a literature survey [26-30]. The levels of urinary polyphenol biomarkers were proved and validated to effectively assess the habitual polyphenol intake and their associations with overweight/obesity in free-living healthy Chinese subjects. Taken together, the identification of biomarkers of dietary polyphenol exposure would provide an additional tool to investigate relationships with chronic disease-related endpoints.

A few previous studies $[14,20,31]$ that related dietary polyphenol intake to metabolic profiles in free-living populations are consistent with our results. Garcia-Perez and colleagues [20] compared urinary metabolic profiles after consumption of different diets and found 19 urinary metabolites to be present in higher concentrations after consumption of healthy diet compared with unhealthy diet. The metabolites reflecting higher intake of fruits and vegetables included hydroxyhippuric acid and hippuric acid, which also showed the capability to distinguish two diets in our study. To the best of our knowledge, this is the first study to evaluate and verify the association between targeted urinary polyphenol biomarkers and polyphenol intake with Chinese dietary habits.

Urine, as a biospecimen, less invasively collected than the blood and more affordable to obtain, offers a greater collection of volumes and thus may turn out to be a potentially highly useful biological resource for large-scale clinical and epidemiologic studies. Ideal biomarkers are reliably measured, specifically exposed, and easily collected [32]. However, owing to the fact that urine metabolite concentrations can be highly variable because of ionic strength, $\mathrm{pH}$, osmolarity, and variations in polyphenol pharmacokinetics, it is sometimes assumed that it may influence the correlations between polyphenol intake and urinary biomarkers [7]. Therefore, in the current study, the selected polyphenols are all of explicit pharmacokinetic characteristics [27], and their absolute concentration in urine is relatively high. Despite the fact that the half-life of some of them is short and the concentration in urine may generally reach the 
TABLE 2: Odds ratios (95\% confidence intervals) for overweight/obesity ${ }^{\mathrm{a}}$.

\begin{tabular}{|c|c|c|c|c|}
\hline & \multicolumn{2}{|c|}{ Univariable } & \multicolumn{2}{|c|}{ Multivariable $^{\mathrm{b}}$} \\
\hline & OR $(95 \% \mathrm{CI})$ & $p$ & OR $(95 \% \mathrm{CI})$ & $p$ \\
\hline Age & $1.034(1.007 \sim 1.062)$ & 0.015 & $1.068(1.016 \sim 1.123)$ & 0.010 \\
\hline Female & $1.347(0.603 \sim 3.011)$ & 0.468 & $1.459(0.239 \sim 8.904)$ & 0.682 \\
\hline FBG & $5.722(2.250 \sim 14.549)$ & $<0.001$ & $4.063(1.299 \sim 12.710)$ & 0.016 \\
\hline UA & $20.619(6.780 \sim 62.707)$ & 0.001 & $1.007(0.998 \sim 1.017)$ & 0.114 \\
\hline CREA & $0.993(0.966 \sim 1.022)$ & 0.646 & $0.992(0.938 \sim 1.050)$ & 0.791 \\
\hline CRP & $1.220(1.014 \sim 1.467)$ & 0.035 & $1.129(0.916 \sim 1.393)$ & 0.255 \\
\hline IsoP & $1.003(0.999 \sim 1.007)$ & 0.143 & $1.002(0.998 \sim 1.007)$ & 0.354 \\
\hline Hypertension $^{\mathrm{a}}$ & $2.394(0.743 \sim 7.714)$ & 0.144 & $0.653(0.132 \sim 3.224)$ & 0.601 \\
\hline Dyslipidemia $^{a}$ & 4.609 (1.782 11.924) & 0.002 & $8.216(2.090 \sim 32.303)$ & 0.003 \\
\hline Current smoker & $0.365(0.100 \sim 1.327)$ & 0.126 & $0.206(0.031 \sim 1.392)$ & 0.105 \\
\hline Current drinker & $0.703(0.256 \sim 1.933)$ & 0.495 & $1.008(0.222 \sim 4.586)$ & 0.992 \\
\hline High levels of physical activity & $0.737(0.324 \sim 1.677)$ & 0.467 & $0.239(0.064 \sim 0.891)$ & 0.033 \\
\hline Polyphenol-rich diet & $1.391(0.600 \sim 3.226)$ & 0.441 & $1.182(0.329 \sim 4.241)$ & 0.798 \\
\hline \multicolumn{5}{|c|}{$z$ score of 22 urinary polyphenol biomarkers as a continuous variable } \\
\hline Per score increment & $0.209(0.056 \sim 0.776)$ & 0.019 & $0.036(0.004 \sim 0.358)$ & 0.005 \\
\hline \multicolumn{5}{|c|}{$z$ score of 22 urinary polyphenol biomarkers as a categorical variable } \\
\hline $\mathrm{T} 1$ & 1.0 (referent) & - & 1.0 (referent) & - \\
\hline $\mathrm{T} 2$ & $1.378(0.555 \sim 3.421)$ & 0.489 & $0.573(0.158 \sim 2.076)$ & 0.397 \\
\hline T3 & $0.369(0.124 \sim 1.095)$ & 0.073 & $0.133(0.027 \sim 0.666)$ & 0.014 \\
\hline$p$ value for trend & 0.090 & & 0.015 & \\
\hline
\end{tabular}

FBG: fasting blood glucose; UA: uric acid; CREA: creatinine; CRP: C-reactive protein; IsoP: 8 -isoprostane. ${ }^{\mathrm{a}}$ Defined according to the criteria of metabolic syndrome from Chinese Diabetes Society (CDS) (2004) for Chinese. 'bet of independent variables: age, gender, FBG, UA, CREA, CRP, IsoP, hypertension, dyslipidemia, smoking and alcohol drinking status, high levels of physical activity, polyphenol-rich diet, and $z$ score of 22 urinary polyphenol biomarkers as a continuous or categorical variable.

TABLE 3: Odds ratios (95\% confidence intervals) of each type of polyphenol biomarkers for overweight/obesity ${ }^{\mathrm{a}}$.

\begin{tabular}{lcc}
\hline$z$ score & Multivariable & \\
& OR $(95 \%$ CI $)$ & $p$ \\
\hline Hydroxycinnamic acids $^{c}$ & $0.291(0.069 \sim 1.223)$ & 0.092 \\
Hydroxybenzoic acids $^{c}$ & $0.576(0.225 \sim 1.473)$ & 0.249 \\
Flavonols $^{c}$ & $0.516(0.149 \sim 1.792)$ & 0.298 \\
Favanones $^{c}$ & $0.461(0.178 \sim 1.193)$ & 0.110 \\
Gut microbial metabolites $^{c}$ & $0.099(0.019 \sim 0.510)$ & 0.006 \\
\hline
\end{tabular}

${ }^{a}$ Defined according to the criteria of metabolic syndrome from Chinese Diabetes Society (CDS) (2004) for Chinese. ${ }^{\mathrm{b}} \mathrm{Set}$ of independent variables: age, gender, FBG, UA, CREA, CRP, IsoP, hypertension, dyslipidemia, smoking and alcohol drinking status, high levels of physical activity, polyphenol-rich diet, and $z$ score of each type of polyphenol biomarkers as a continuous variable. ${ }^{\text {cT }}$ The $z$ score of each type of polyphenol biomarkers was computed by the summary of the $z$ score of each polyphenol biomarker/numbers of biomarkers of each type.

basic level in $48 \mathrm{~h}$, some studies demonstrated that the concentration in urine may possibly achieve a detectable stable state when people intake polyphenol-rich foods daily on a regular basis [7, 28, 33].

The inverse associations between levels of urinary polyphenol biomarkers and overweight/obesity were also observed after being controlled for multivariates. ROC curves

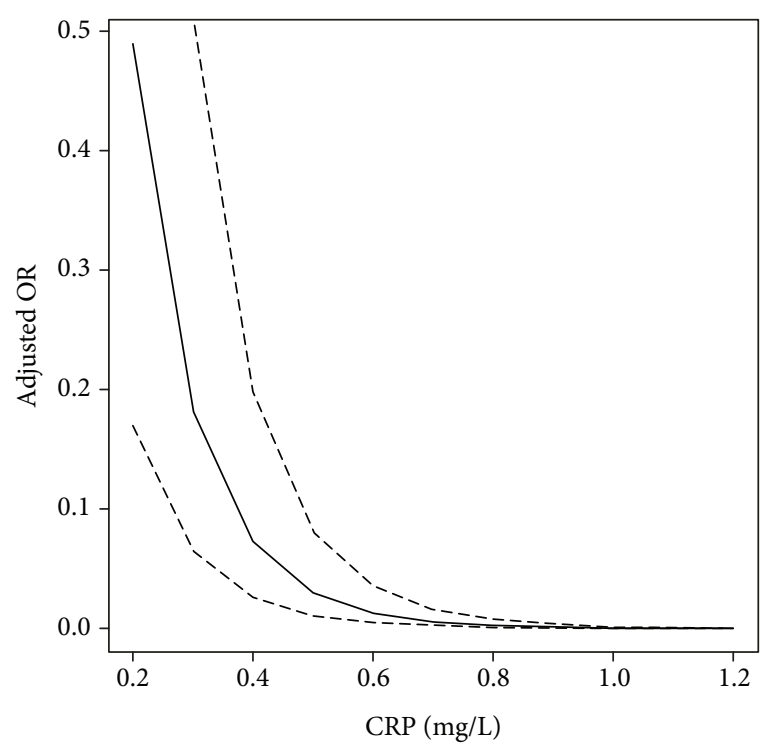

FIGURe 4: Adjusted odds ratios (OR) of levels of urinary polyphenol biomarkers for overweight/obesity by serum CRP concentrations. Serum CRP concentrations were modeled as restricted cubic splines with three freedom degrees. Multivariable logistic regression models (for overweight/obesity) were adjusted for age (continuous) and gender. The $z$ score of urinary polyphenol biomarkers at the 25th percentile was used as reference. The dashed lines represent the $95 \%$ confidence intervals. 


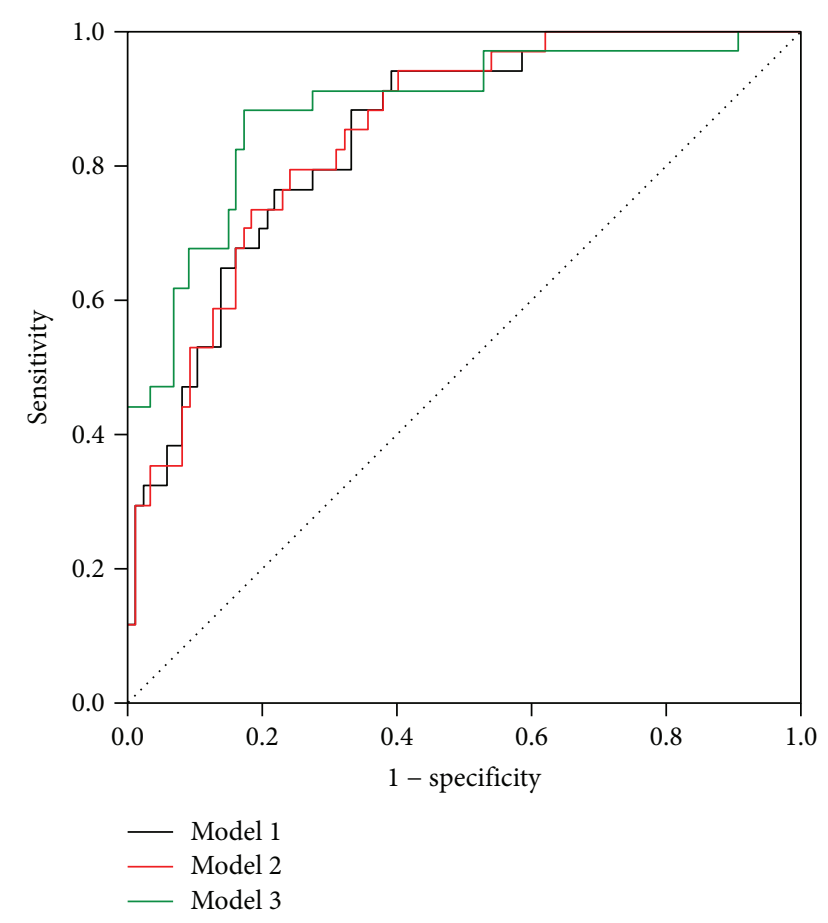

FIGURE 5: Receiver operating characteristic (ROC) curves for overweight/obesity. ROC curves and measures of the area under the curve (AUC) are presented for three multivariable models: (1) clinical model inclusive of traditional risk factors (age, gender, FBG, UA, CREA, CRP, IsoP, hypertension, dyslipidemia, smoking and alcohol drinking status, and physical activity; black solid line); (2) model 1 plus polyphenol-rich diet (red line); and (3) model 2 plus the $z$ score of 22 urinary polyphenol biomarkers (green line).

to assess the performance of the classifier models for overweight/obesity classification showed that using biomarkers in combination with self-reports could strengthen the discriminative capability of the clinical model. Through analyzing the relationship of each type of polyphenol biomarkers with overweight/obesity, we observed that urinary levels of gut microbial metabolites of polyphenol had the most significant and negative association with overweight/obesity. So far as we know, current thinking considers gut microbiota playing a potential role on the development of obesity and its related comorbidities [34]. And dietary habits are considered to be the main contributing factors to the diversity of the human gut microbiota by altering its composition [35]. A number of studies have shown long-term dietary patterns and short-term interventions induce changes in gut microbiota structure and function, and they have also revealed the fact that metabolic changes ultimately could influence host physiological health [36]. Our findings support these points of views on the relationship among diet, gut microbiota, and obesity.

The microbial polyphenol metabolism is kept to a general pattern, in which the extremely diverse group of plant polyphenols is transformed into a relatively small number of common metabolites [37]. Because of the complex determination of fecal flora, there is a need for rapid and sensitive analytical methods that can quantify such metabolites for a large number of samples with rapid and sensitive targeted metabolomics analysis [38]. A growing number of studies have shown concern on the determination of polyphenol microbial metabolites, especially in urine or plasma, but only a few have been committed to their accurate quantification in different biospecimens [39]. The developed method in the current study has brought about a possibility of using the simple method for quantifying or evaluating gut microbiota and their association with diet and disease or their interaction in the future.

In addition, a significant interaction was observed between urinary polyphenol biomarker levels and CRP for overweight/obesity. CRP is a marker of chronic inflammation and is an independent predictor of all-cause cardiovascular mortality. The negative association of urinary polyphenol biomarker levels with overweight/obesity was more pronounced in the inflammatory groups in the present study. What's more, with the increase of serum CRP concentration, the inverse relationship of polyphenol biomarkers with overweight/obesity gradually enhanced. Those consistent findings showed that the association of urinary polyphenol biomarker levels with overweight/obesity was more pronounced in participants with a chronic inflammatory status than others. The chronic low-grade inflammatory condition accompanying obesity has been implicated as a major player in both obesity and its associated pathophysiological consequences [40]. Thus, the current results demonstrate that it is of crucial importance to maintain polyphenol biomarkers at high levels or intakesufficient polyphenols in obesity with chronic inflammation than others.

There are several limitations. Firstly, results were based on a short and simple self-reported FFQ that may likely attenuate the observed correlations. The FFQ provided information on the frequency of consumption of most common polyphenol-rich foods in Chinese dietary habit, but exclusive of weight and portion size, because there is no need to estimate the mean daily polyphenol intake as a continuous variable for the lack of a Chinese-specific food polyphenol composition database. However, it is because of the reasons mentioned above; our results proved that urinary polyphenol biomarkers could offer a valid alternative or complementary addition to self-reported survey for the evaluation of polyphenol intake while it is impossible for large-scale epidemiologic studies to collect details of dietary information. Secondly, metabolite concentrations account for the genetic effect modification of metabolic processes. We were unable to evaluate that, which could influence the extent to which a metabolite can act as a universal dietary biomarker. Thirdly, we cannot rule out the possibility that some findings may have been derived by chance, but we attempted to minimize this by controlling for multivariates. Finally, except for overweight/obese, there are no statistical differences of levels of urinary polyphenol biomarkers between different groups according to the presence of other components of MetS. The limitations of the cross-sectional study design and small sample size may account for this discrepancy. The findings need to be validated in future studies. 


\section{Conclusions}

A targeted metabolomics method by LC/MS/MS for the measurement of 22 polyphenol biomarkers in urine samples was established and validated to effectively assess the habitual polyphenol intake in free-living healthy Chinese subjects. We also found that the levels of urinary polyphenol biomarkers, especially gut microbial metabolites of polyphenols, were inversely associated with overweight and obesity, and this association was more pronounced in the inflammatory groups suggesting that it is of great importance to maintain polyphenol biomarkers at high levels or intake-sufficient polyphenols in obesity with chronic inflammation than others. The measurement of these biomarkers may offer a valid alternative or complementary addition to self-reported survey for the evaluation of polyphenol intake and investigation into their relationships with chronic disease-related endpoints in large-scale clinical and epidemiologic studies.

\section{Data Availability}

The datasets used and/or analyzed during the current study are available from the corresponding authors on reasonable request.

\section{Conflicts of Interest}

The authors declare that there is no conflict of interest regarding the publication of this paper.

\section{Acknowledgments}

This work was supported by CAMS Innovation Fund for Medical Sciences (grant number 2018-I2M-1-002), the National Natural Science Foundation of China (grant numbers 81301487, 81672075, and 81472035), Beijing Hospital Nova Project (grant number BJ-2018-135), and Beijing Hospital Doctor Foundation Project (grant number BJ2018-025). All authors are grateful to the volunteers of the study and Beijing Hospital for the support given to the data collection.

\section{Supplementary Materials}

Supplementary Table 1: ion transitions for polyphenol biomarkers and instrumental parameters for their MS/MS detection in an MRM mode. (Supplementary Materials)

\section{References}

[1] C. Manach, A. Scalbert, C. Morand, C. Remesy, and L. Jimenez, "Polyphenols: food sources and bioavailability," The American Journal of Clinical Nutrition, vol. 79, no. 5, pp. 727-747, 2004.

[2] D. Vauzour, A. Rodriguez-Mateos, G. Corona, M. J. OrunaConcha, and J. P. E. Spencer, "Polyphenols and human health: prevention of disease and mechanisms of action," Nutrients, vol. 2, no. 11, pp. 1106-1131, 2010.

[3] M. Kampa, A. P. Nifli, G. Notas, and E. Castanas, "Polyphenols and cancer cell growth," in Reviews of Physiology, Biochemistry and Pharmacology, pp. 79-113, Springer, Berlin, Heidelberg, 2007.

[4] L. H. Yao, Y. M. Jiang, J. Shi et al., "Flavonoids in food and their health benefits," Plant Foods for Human Nutrition, vol. 59, no. 3, pp. 113-122, 2004.

[5] A. Satija, E. Yu, W. C. Willett, and F. B. Hu, "Understanding nutritional epidemiology and its role in policy," Advances in Nutrition, vol. 6, no. 1, pp. 5-18, 2015.

[6] D. B. Haytowitz, S. Bhagwat, and J. M. Holden, "Sources of variability in the flavonoid content of foods," Procedia Food Science, vol. 2, pp. 46-51, 2013.

[7] R. Zamora-Ros, M. Rabassa, R. Llorach, C. A. Gonzalez, and C. Andres-Lacueva, "Application of dietary phenolic biomarkers in epidemiology: past, present, and future," Journal of Agricultural and Food Chemistry, vol. 60, no. 27, pp. 6648-6657, 2012.

[8] A. Schatzkin, A. F. Subar, S. Moore et al., "Observational epidemiologic studies of nutrition and cancer: the next generation (with better observation)," Cancer Epidemiology Biomarkers of Prevention, vol. 18, no. 4, pp. 1026-1032, 2009.

[9] R. H. Keogh and I. R. White, "A toolkit for measurement error correction, with a focus on nutritional epidemiology," Statistics in Medicine, vol. 33, no. 12, pp. 2137-2155, 2014.

[10] K. L. Rennie, A. Coward, and S. A. Jebb, "Estimating underreporting of energy intake in dietary surveys using an individualised method," British Journal of Nutrition, vol. 97, no. 6, pp. 1169-1176, 2007.

[11] M. Jenab, N. Slimani, M. Bictash, P. Ferrari, and S. A. Bingham, "Biomarkers in nutritional epidemiology: applications, needs and new horizons," Human Genetics, vol. 125, no. 5-6, pp. 507-525, 2009.

[12] N. Potischman, "Biologic and methodologic issues for nutritional biomarkers," The Journal of Nutrition, vol. 133, no. 3, pp. 875s-880s, 2003.

[13] L. S. Freedman, N. Tasevska, V. Kipnis et al., "Gains in statistical power from using a dietary biomarker in combination with self-reported intake to strengthen the analysis of a dietdisease association: an example from CAREDS," American Journal of Epidemiology, vol. 172, no. 7, pp. 836-842, 2010.

[14] K. A. Guertin, S. C. Moore, J. N. Sampson et al., "Metabolomics in nutritional epidemiology: identifying metabolites associated with diet and quantifying their potential to uncover diet-disease relations in populations," The American Journal of Clinical Nutrition, vol. 100, no. 1, pp. 208-217, 2014.

[15] D. P. Jones, Y. Park, and T. R. Ziegler, "Nutritional metabolomics: progress in addressing complexity in diet and health," Annual Review of Nutrition, vol. 32, no. 1, pp. 183202, 2012.

[16] M. Beckmann, A. J. Lloyd, S. Haldar et al., "Dietary exposure biomarker-lead discovery based on metabolomics analysis of urine samples," Proceedings of the Nutrition Society, vol. 72, no. 3, pp. 352-361, 2013.

[17] A. J. Lloyd, M. Beckmann, S. Haldar, C. Seal, K. Brandt, and J. Draper, "Data-driven strategy for the discovery of potential urinary biomarkers of habitual dietary exposure," The American Journal of Clinical Nutrition, vol. 97, no. 2, pp. 377-389, 2013.

[18] A. O'Sullivan, M. J. Gibney, and L. Brennan, "Dietary intake patterns are reflected in metabolomic profiles: potential role in dietary assessment studies," The American Journal of Clinical Nutrition, vol. 93, no. 2, pp. 314-321, 2011. 
[19] E. Pujos-Guillot, J. Hubert, J. F. Martin et al., "Mass spectrometry-based metabolomics for the discovery of biomarkers of fruit and vegetable intake: citrus fruit as a case study," Journal of Proteome Research, vol. 12, no. 4, pp. 1645-1659, 2013.

[20] I. Garcia-Perez, J. M. Posma, R. Gibson et al., "Objective assessment of dietary patterns by use of metabolic phenotyping: a randomised, controlled, crossover trial," The Lancet Diabetes \& Endocrinology, vol. 5, no. 3, pp. 184-195, 2017.

[21] A. Medina-Remón, A. Barrionuevo-González, R. Zamora-Ros et al., "Rapid Folin-Ciocalteu method using microtiter 96-well plate cartridges for solid phase extraction to assess urinary total phenolic compounds, as a biomarker of total polyphenols intake," Analytica Chimica Acta, vol. 634, no. 1, pp. 54-60, 2009.

[22] V. Neveu, J. Perez-Jimenez, F. Vos et al., "Phenol-Explorer: an online comprehensive database on polyphenol contents in foods," Database, vol. 2010, article bap024, 2010.

[23] Research Collaboration Group of Metabolic Syndrome of Chinese Diabetes Society, "Suggestions of Chinese Diabetes Society (CDS) for the metabolic syndrome," Chinese Journal of Diabetes, vol. 12, no. 3, pp. 156-161, 2004.

[24] J. S. Yudkin, C. D. A. Stehouwer, J. J. Emeis, and S. W. Coppack, "C-reactive protein in healthy subjects: associations with obesity, insulin resistance, and endothelial dysfunction: a potential role for cytokines originating from adipose tissue?," Arteriosclerosis, Thrombosis, and Vascular Biology, vol. 19, no. 4, pp. 972-978, 1999.

[25] Z. Yu, X. Ye, J. Wang et al., "Associations of physical activity with inflammatory factors, adipocytokines, and metabolic syndrome in middle-aged and older Chinese people," Circulation, vol. 119, no. 23, pp. 2969-2977, 2009.

[26] J. Pérez-Jiménez, J. Hubert, L. Hooper et al., "Urinary metabolites as biomarkers of polyphenol intake in humans: a systematic review," The American Journal of Clinical Nutrition, vol. 92, no. 4, pp. 801-809, 2010.

[27] C. Manach, G. Williamson, C. Morand, A. Scalbert, and C. Remesy, "Bioavailability and bioefficacy of polyphenols in humans. I. Review of 97 bioavailability studies," The American Journal of Clinical Nutrition, vol. 81, no. 1, pp. 230s-242s, 2005.

[28] S. M. Henning, P. Wang, N. Abgaryan et al., "Phenolic acid concentrations in plasma and urine from men consuming green or black tea and potential chemopreventive properties for colon cancer," Molecular Nutrition \& Food Research, vol. 57, no. 3, pp. 483-493, 2013.

[29] L. I. Mennen, D. Sapinho, H. Ito et al., "Urinary flavonoids and phenolic acids as biomarkers of intake for polyphenol-rich foods," British Journal of Nutrition, vol. 96, no. 01, pp. 191198, 2006.

[30] W. M. Loke, A. M. Jenner, J. M. Proudfoot et al., “A metabolite profiling approach to identify biomarkers of flavonoid intake in humans," The Journal of Nutrition, vol. 139, no. 12, pp. 2309-2314, 2009.

[31] M. C. Playdon, J. N. Sampson, A. J. Cross et al., "Comparing metabolite profiles of habitual diet in serum and urine," The American Journal of Clinical Nutrition, vol. 104, no. 3, pp. 776-789, 2016.
[32] F. Gil and A. Pla, "Biomarkers as biological indicators of xenobiotic exposure," Journal of Applied Toxicology, vol. 21, no. 4, pp. 245-255, 2001.

[33] M. D’Archivio, C. Filesi, R. Varì, B. Scazzocchio, and R. Masella, "Bioavailability of the polyphenols: status and controversies," International Journal of Molecular Sciences, vol. 11, no. 4, pp. 1321-1342, 2010.

[34] L. Zhao, "The gut microbiota and obesity: from correlation to causality," Nature Reviews Microbiology, vol. 11, no. 9, pp. 639-647, 2013.

[35] K. Al-Assal, A. C. Martinez, R. S. Torrinhas, C. Cardinelli, and D. Waitzberg, "Gut microbiota and obesity," Clinical Nutrition Experimental, vol. 20, pp. 60-64, 2018.

[36] K. J. Portune, A. Benítez-Páez, E. M. G. del Pulgar, V. Cerrudo, and Y. Sanz, "Gut microbiota, diet, and obesity-related disorders-The good, the bad, and the future challenges," Molecular Nutrition \& Food Research, vol. 61, no. 1, 2017.

[37] E. P. Nyangale, D. S. Mottram, and G. R. Gibson, "Gut microbial activity, implications for health and disease: the potential role of metabolite analysis," Journal of Proteome Research, vol. 11, no. 12, pp. 5573-5585, 2012.

[38] A. Scalbert, L. Brennan, O. Fiehn et al., "Mass-spectrometrybased metabolomics: limitations and recommendations for future progress with particular focus on nutrition research," Metabolomics, vol. 5, no. 4, pp. 435-458, 2009.

[39] M. Gasperotti, D. Masuero, G. Guella, F. Mattivi, and U. Vrhovsek, "Development of a targeted method for twenty-three metabolites related to polyphenol gut microbial metabolism in biological samples, using SPE and UHPLCESI-MS/MS,” Talanta, vol. 128, pp. 221-230, 2014.

[40] R. Monteiro and I. Azevedo, "Chronic inflammation in obesity and the metabolic syndrome," Mediators of Inflammation, vol. 2010, Article ID 289645, 10 pages, 2010. 


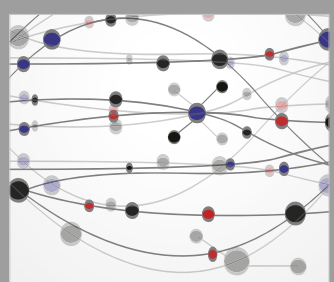

The Scientific World Journal
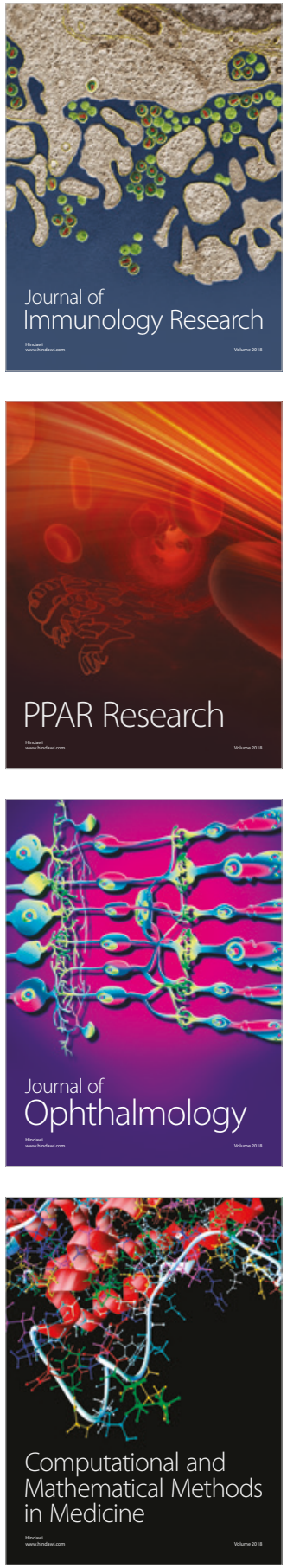

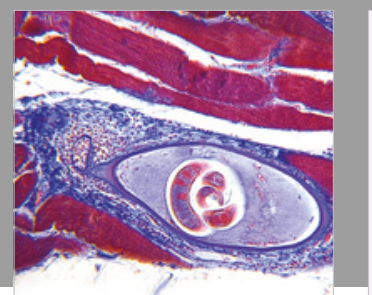

Gastroenterology Research and Practice

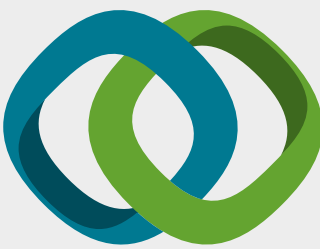

\section{Hindawi}

Submit your manuscripts at

www.hindawi.com
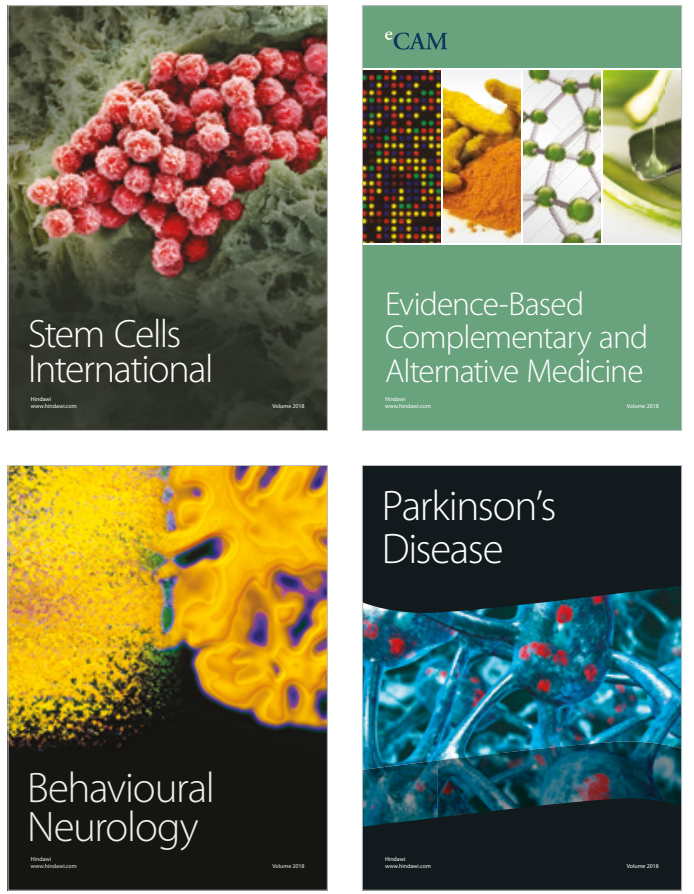

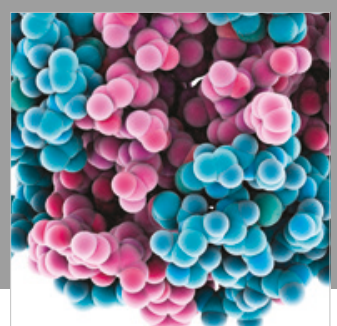

ournal of

Diabetes Research

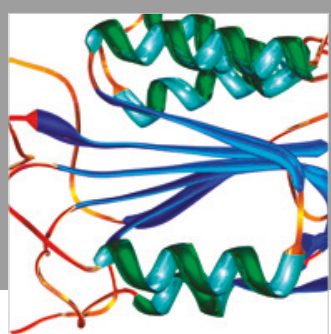

Disease Markers
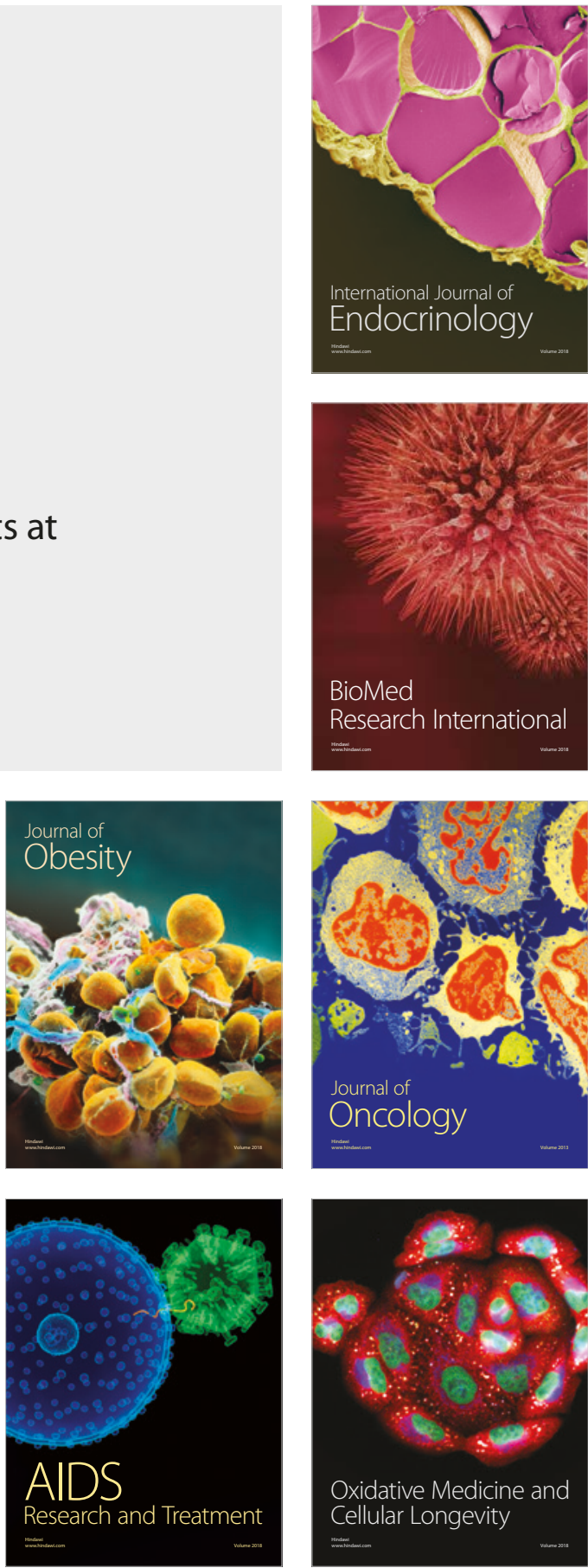